\title{
Suchtartiges Arbeiten: Persönlichkeitsfaktoren und berufliche Rahmenbedingungen
}

\author{
Maximilian Pannier und Mira Fauth-Bühler 우 \\ Institut für Wirtschaftspsychologie (iwp), FOM Hochschule für Oekonomie \& Management, Hochschulzentrum Stuttgart
}

\begin{abstract}
Zusammenfassung: Fragestellung: Suchtartiges Arbeiten stellt ein ernst zu nehmendes Problem in unserer Gesellschaft dar. In dieser Studie wird der Frage nachgegangen, welche Rolle berufliche Rahmenbedingungen (Entgrenzung der Arbeit) und Persönlichkeitsfaktoren (Big Five) bei suchtartigem Arbeiten spielen. Methode: 111 Erwerbstätige im Alter von 19 bis 66 Jahren beantworteten online den Persönlichkeitstest Big Five Inventory-SOEP, den Fragebogen zur Erfassung zentraler Merkmale der Arbeit 4.0 und die Skala zur Erfassung von Arbeitssucht. Die statistischen Auswertungen (Korrelationsanalysen, moderierte Regressionsanalyse) erfolgten mittels „R“ und „PSPP“. Ergebnisse: Suchtartiges Arbeiten korreliert signifikant mit Entgrenzung $(r=.208)$, Neurotizismus $(r=.356)$ und dem Alter der Probanden $(r=-.321)$. Alle drei Prädiktoren zusammen erklären insgesamt 29 Prozent der Varianz von suchtartigem Arbeiten. Es konnte kein moderierender Effekt der Variablen Entgrenzung der Arbeit auf den Zusammenhang zwischen Neurotizismus und suchtartigem Arbeiten gefunden werden. Schlussfolgerungen: Neben dem Persönlichkeitsfaktor Neurotizismus und dem Alter spielen bei der Vorhersage suchtartigen Arbeitens auch das Ausmaß der Entgrenzung der Arbeit eine relevante Rolle. Die Ergebnisse sind vor dem Hintergrund der Covid-19-Pandemie relevant, da bei Mitarbeitern im Homeoffice die Grenzen von Arbeit und Privatleben besonders stark verwischen.
\end{abstract}

Schlüsselwörter: Suchtartiges Arbeiten, Persönlichkeit, Neurotizismus, berufliche Rahmenbedingungen, Verhaltenssüchte

Workaholism: Personality Factors and Conditions of Professional Work

\begin{abstract}
Question: The need for excessive work, also known as “workaholism”, is a serious health problem in our society. In this study, we examined the impact of work characteristics (low boundaries between work and home) and personality traits (Big Five) on workaholism. Method: Data were collected from a sample of 111 employees aged 19 to 66. Participants completed the Big Five Inventory-SOEP (BFI-S), a questionnaire to determine central characteristics of work (Fragebogen zur Erfassung der zentralen Merkmale der Arbeit 4.0) and a scale to measure workaholism (Skala zur Erfassung von Arbeitssucht) online. Statistical analyses (correlation analyses, moderated regression analyses) were conducted using "R" and "PSPP". Results: Workaholism scores were found to be significantly correlated with low boundaries between work and home $(r=.208)$, with neuroticism $(r=.356)$ and with participants' age $(r=-.321)$. The proportion of variance explained by all three predictors was $29 \%$. No moderating effect of the variable 'boundaries between work and home' on the relationship between neuroticism (UV) and workaholism (AV) could be found. Conclusions: In addition to the Big Five personality factor neuroticism and the age of participants, low boundaries between work and home play a relevant role in workaholism. These results are important in the context of the COVID-19 pandemic, as employees more often work from home, which makes the separation between work and personal time more difficult.
\end{abstract}

Keywords: Workaholism, personality, neuroticism, work characteristics, behavioural addictions

\section{Einleitung}

Schon in den späten 1950er Jahren wurde die sogenannte "Arbeitssucht" als Managerkrankheit in populären Medien aufgegriffen (Der Spiegel, 1958). Das lag daran, dass zu dieser Zeit der Herzinfarkt als Todesursache in der Bevölkerung deutlich stieg. Managern, in deren Berufsgruppe Stress, hohe Arbeitsdichte und Verantwortung als Teil der Tätigkeit galt, waren besonders häufig betroffen. Daher sind auch in frühen Studien zu suchtartigem Arbeiten überwiegend Manager untersucht worden (Rademacher,
2017). Spätere Untersuchungen konnten aber zeigen, dass suchtartiges Arbeiten jeden, unabhängig von Geschlecht, Alter und Berufsstatus, gleichermaßen treffen kann (Städele \& Poppelreuter, 2009). Obwohl die Prävalenz der Arbeitssucht, aufgrund von fehlender klinischer Diagnosekriterien, schwer zu bestimmen ist, zeigt sich dennoch eine steigende Anzahl von Erkrankten (Andreassen et al., 2016). Mit steigender medialer Präsenz und fortschreitender Entwicklung in der Forschung, bildeten sich zunehmend Selbsthilfegruppen. Auch Rehabilitationskliniken nahmen arbeitssüchtige Personen auf, und zwar trotz 
der fehlenden Anerkennung als psychische Störung (Städele \& Poppelreuter, 2009). Hinzu kommt, dass die moderne Leistungsgesellschaft der Arbeit einen hohen Stellenwert beimisst. Ein Individuum erhält durch die Arbeit Bedeutung in der Gesellschaft (Weimar, Braakmann, Gelo \& Schiava-Winkler, 2009). In diesem Punkt unterscheidet sich die Arbeitssucht von allen klassischen substanzgebundenen Süchten und auch von vielen Verhaltenssüchten. Während die klassischen Süchte häufig sozial geächtet werden, vollbringt die arbeitssüchtige Person eine Tätigkeit, die ihr soziale Anerkennung einbringt (Rademacher, 2017). Dieser Widerspruch wird auch in der frühen Forschungsliteratur sichtbar, in der auf der einen Seite suchtartiges Arbeiten als erstrebenswert galt (Machlowitz, 1981) und auf der anderen Seite nachdrücklich aufgrund der negativen Folgen davor gewarnt wurde (Fassel, 1991). Daher ist es umso wichtiger, die klinische Relevanz des Themas in den Fokus der Forschung zu rücken. Denn heute ist sich die Forschung zu großen Teilen einig, dass arbeitssüchtiges Verhalten negative Folgen für das Individuum, sein soziales Umfeld und auch für das Unternehmen, in dem es beschäftigt ist, hat (Andreassen et al., 2013; Bakker, Demerouti \& Burke, 2009; Griffiths, 2011; Rademacher, 2017; Städele \& Poppelreuter, 2009). Erschwerend für die Erforschung von suchtartigem Arbeitsverhalten kommt hinzu, dass es an einheitlichen Diagnosekriterien mangelt. Der Begriff „workaholism“ wurde von Oates im Jahr 1971 in Anlehnung an den Begriff „alcoholism“ geprägt und im deutschsprachigen Raum sprach Mentzel 1979, also 8 Jahre später, erstmals von „Arbeitssucht“. Neben frühen Arbeiten zum Thema suchtartiges Arbeiten, erlebt das Thema derzeit wieder eine Renaissance. Im Zuge der Erweiterung der Diagnosesysteme um die Kategorie der Verhaltenssüchte rückt auch das Thema suchtartiges Arbeiten wieder verstärkt in den Fokus. Ob es sich dabei um eine Suchterkrankung handelt, ist gegenwärtig Diskussionsgegenstand. Während manche Wissenschaftler vor einem inflationären Gebrauch des Suchtbegriffes und einer Pathologisierung der Gesellschaft warnen (z. B. Starcevic, V., Billieux, J. \& Schimmenti, A., 2018; Billieux et al., 2015; Brand et al., 2020 ), wird in anderen Arbeiten eine Definition von suchtartigem Arbeiten in Anlehnung an die klassischen Suchtkriterien verfolgt (z.B. Loscalzo \& Giannini, 2017; Griffiths, Demetrovics \& Atroszko, 2018). Die Definition von exzessivem Arbeiten als Verhaltenssucht ist methodisch bisher die einzige Definition, die auf einer diagnostischen und ausführlichen theoriebasierten Grundlage beruht und ist folglich zum jetzigen Zeitpunkt methodisch den anderen Definitionen vorzuziehen.

Bisherige Forschungsbefunde haben substanzgebundene als auch substanzungebundene Süchte mit Veränderungen in der Persönlichkeit in Verbindung gebracht (z.B.
Mann et al., 2017; Zilberman et al., 2018). Innerhalb des fünf-Faktoren Modells der Eigenschaftstheorien zeigte sich, dass Neurotizismus der Persönlichkeitsfaktor ist, der am häufigsten mit substanzgebundenen und mit substanzungebundenen Süchten einhergeht. Hier konnten sich Zusammenhänge mit Sucht allgemein (Homayouni, 2011), mit Computerspielsucht (Mehroof \& Griffiths, 2010), mit Internetsucht (Senormanci et al., 2014; Andreassen et al., 2013) und auch mit Arbeitssucht (Burke, Matthiesen \& Pallessen, 2006; Součková, Vaculík \& Procházka, 2014) finden. Eine Studie, die die Arbeitssucht nah an den klassischen Süchten definierte, konnte zeigen, dass Neurotizismus einen signifikanten Zusammenhang mit suchtartigem Arbeiten aufweist (Andreassen et al., 2013). Des Weiteren neigen Personen mit hohen Werten im Faktor Neurotizismus zu Selbstzweifeln (Neyer \& Asendorpf, 2018). Im Vergleich dazu kann übermäßiges Arbeiten dazu dienen, ein verletztes oder geschwächtes Selbstwertgefühl zu stärken (Meißner, 2005). Somit könnte ein Zusammenhang zwischen Neurotizismus und suchtartigem Arbeiten dadurch erklärt werden, dass Personen mit hohen Werten im Faktor Neurotizismus durch eine übermäßige Arbeitsanstrengung versuchen, ihr schlechtes Selbstwertgefühl auszugleichen. Zwar kommen Studien auch zu gegensätzlichen Ergebnissen (Sharma \& Sharma, 2011; Aziz \& Tronzo, 2011), jedoch wurden hier andere Fragebögen eingesetzt, in denen exzessives Arbeiten unterschiedlich konzeptionalisiert wurde. Berücksichtigung fanden dort u.a. Aspekte wie Freude am Arbeiten oder zwanghafte Aspekte. Da die vorliegende Arbeit aber suchtartiges Arbeiten stark an den klassischen Süchten definiert, wird postuliert, dass Neurotizismus in der vorliegenden Stichprobe einen positiven Zusammenhang mit suchtartigem Arbeiten vorweist. Deshalb lautet die Hypothese 1 (H1): „Der Persönlichkeitsfaktor Neurotizismus steht in positivem Zusammenhang mit suchtartigem Arbeiten."

Unter der Entgrenzung wird die zunehmende Dynamisierung der bestehenden (begrenzenden) Strukturen der Arbeit bezeichnet, welche lange durch tayloristisch und fordistisch geprägte Ansätze dominiert waren. Im Fordismus werden die angestrebten Ziele mit Hilfe von technischer Kontrolle des Arbeitsprozesses erreicht und im Taylorismus geschieht dies mit Hilfe von organisatorischen Mitteln. Durch die Entgrenzung der Arbeit wird nun die Verantwortlichkeit der Arbeitnehmer erhöht, mit dem Ziel, neben der Kosteneinsparung neue Flexibilitäts- und Innovationspotentiale freizusetzen (Kleemann \& Voß, 2018). Konkret bedeutet dies, dass Beschäftigte vermehrt an ihrem Output, also ihrer tatsächlichen Leistung, statt ihrer Arbeitszeit gemessen werden. Das bedeutet nicht zwangsläufig, dass die Vergütung auf dem Output basiert, sondern dass Kontrollinstanzen vermehrt auf die erbrachten Ergebnisse statt auf die geleistete Arbeitszeit achten 
(Poethke, Klasmeier, Diebig, Hartmann \& Rowold, 2019). Diese Veränderungen sind ambivalent zu betrachten: Was für die einen eine deutliche Verbesserung der Arbeitsbedingungen bedeuten kann, ist für die anderen eine Verschlechterung. Zum Beispiel kann eine erhöhte Selbstverantwortung auch zu einer Selbstüberforderung führen, wenn die verfügbaren Ressourcen nicht ausreichen, um die steigenden Anforderungen zu bewältigen (Böhle, 2018). Des Weiteren kann die zunehmende Übertragung von Verantwortung auf Beschäftigte, speziell im agilen Umfeld, diese überfordern, sofern Sie das Bedürfnis nach fester Struktur und Vorgaben besitzen (Rump \& Eilers, 2017). Mit dem Ausbruch der Corona-Pandemie spielt das Problem der Entgrenzung bei immer mehr Arbeitnehmern eine Rolle, da die Arbeit von immer mehr Menschen im Homeoffice durchgeführt wird und Vorgesetzte mehr auf die erbrachten Ergebnisse statt auf die geleistete Arbeitszeit achten (Möhring et al., 2020).

Da Beschäftigte durch die Entgrenzung der Arbeit zunehmend an ihrem Output gemessen werden, wird angenommen, dass sich außerhalb der regulären Arbeitszeit die Beschäftigungsdauer erhöht (Poethke et al., 2019). Dies führt zu einer Ausdehnung der Arbeitszeit insgesamt, da es Personen gibt, die die vorgegebenen Ziele in der regulären Arbeitszeit nicht schaffen. Zusätzlich führt es dazu, dass sich Betroffene schwerer von der Arbeit lösen können, was wiederum zu einer Erholungsunfähigkeit führen kann, da auch in der Freizeit viel über die Arbeit sinniert wird (Hassler, Rau, Hupfeld \& Paridon, 2013). Suchtartiges Arbeiten ist ebenfalls durch eine fehlende gedankliche Distanzierung von der Arbeit charakterisiert (Hägerbäumer, 2017). Es wird angenommen, dass ein positiver Zusammenhang zwischen Entgrenzung und suchtartigem Arbeiten besteht. Darum lautet die Hypothese 2 (H2): ,Eine zunehmend entgrenzte Arbeit steht in positivem Zusammenhang mit suchtartigem Arbeiten."

Bisher gibt es keine Untersuchungen, die eine Kombination aus Rahmenbedingungen und Persönlichkeit in den Fokus zur Erklärung von suchtartigem Arbeiten stellen. Studien untersuchten meist nur eines der beiden Konstrukte in Verbindung mit Arbeitssucht (Lior, Abira \& Aviv, 2018). Bisherige Untersuchungen zeigen, dass bestimmte Rahmenbedingungen für bestimmte Personen gut und für andere schlecht sind (Böhle, 2018). Doch worin sich diese Personen unterscheiden, wurde noch nicht genau untersucht. Darum wird hier postuliert, dass eine Entgrenzung der Arbeit für diejenigen eine Bereicherung der Arbeit darstellt, die über geringe Ausprägungen im Persönlichkeitsfaktor Neurotizismus verfügen. Nicht-ängstliche Menschen, die eigenverantwortlich arbeiten können, erleben ein höheres Involvement in Ihre Arbeit und erleben Autonomie und Kontrolle. Ängstliche Menschen sind schneller gestresst und halten diesen Stresslevel auch länger (Ney- er \& Asendorpf, 2018). Somit arbeiten sie mehr, um die vorgegeben Ziele zu erreichen, da sie stärker befürchten, diese zu verfehlen. Darum lautet die Hypothese 3 (H3): „Der Zusammenhang zwischen dem Persönlichkeitsfaktor Neurotizismus und suchtartigem Arbeiten wird vom Ausmaß der Entgrenzung der Arbeit beeinflusst."

\section{Methodik}

\section{Stichprobe und Durchführung}

Insgesamt nahmen 144 Personen an der Erhebung teil. Von diesen waren 22 nicht erwerbstätig und weitere elf Personen füllten den Fragebogen nicht vollständig aus. Somit verblieb eine Stichprobe von 111 Personen. Die Teilnehmer waren im Durchschnitt 30.8 Jahre alt (SD: 11.46), $37,8 \%$ waren männlich, $62,2 \%$ waren weiblich und $91,9 \%$ in einem Angestelltenverhältnis mit unterschiedlichen Arbeitszeitmodellen beschäftigt. Alle soziodemographischen Daten sind in Tabelle 1 zusammengefasst.

Die Online-Erhebung fand zwischen dem 27.09. und 21.10.2019 statt. Die Internetseite umfrageonline.com wurde als Plattform für die Online Erhebung verwendet. Die Rekrutierung der Teilnehmer erfolgte über die Versendung von Teilnahmelinks via E-Mail und Social Media. In der ersten Welle der Teilnehmerakquise wurden WhatsApp Kontakte des Autors kontaktiert, in der zweiten Welle wurden Mitglieder von Facebook akquiriert und in der dritten Welle wurden Studierende via E-Mail gebeten, an der Befragung teilzunehmen.

Nach den soziodemographischen Fragen wurden den Teilnehmern zuerst der Fragebogen zur Erfassung zentraler Merkmale der Arbeit 4.0 von Poethke, Klasmeier, Diebig, Hartmann und Rowold (2019), gefolgt von der Skala zur Erfassung von Arbeitssucht von Schneider und Bühler (2014) und zuletzt der Big-Five-Inventory-SOEP (BFI-S) von Schupp \& Gerlitz (2014) zur Beantwortung vorgelegt.

\section{Instrumente und Auswertung}

Um das Konstrukt „Entgrenzung der Arbeit“ zu erheben, kam der Fragebogen zur Erfassung zentraler Merkmale der Arbeit 4.0 von Poethke, Klasmeier, Diebig, Hartmann und Rowold (2019) zum Einsatz. Für die in dieser Arbeit gewählte Fragestellung wurde die Dimension Entgrenzung der Arbeitstätigkeit verwendet, da die Literatur überwiegend für diesen Punkt eine zusätzliche Steigerung der beruflichen Anforderungen gezeigt hat (Böhle, 2018). Um diese Dimension zu erfassen, werden je fünf Statements, bspw. „Ich bin im Urlaub jederzeit für meine Kollegen und 
Tabelle 1. Soziodemographische Daten der Stichprobe

\begin{tabular}{|c|c|c|}
\hline Variable & $\mathrm{n}$ & $\%$ \\
\hline \multicolumn{3}{|l|}{ Geschlecht } \\
\hline Männlich & 42 & 37,8 \\
\hline Weiblich & 69 & 62,2 \\
\hline Divers & 0 & 0 \\
\hline \multicolumn{3}{|l|}{ Bundesland } \\
\hline Baden-Württemberg & 58 & 52,3 \\
\hline Nordrhein-Westfalen & 21 & 18,9 \\
\hline Bayern & 8 & 7,2 \\
\hline Hessen & 8 & 7,2 \\
\hline Niedersachsen & 5 & 4,5 \\
\hline Berlin & 4 & 3,6 \\
\hline Rheinland-Pfalz & 3 & 2,7 \\
\hline Brandenburg & 2 & 1,8 \\
\hline Hamburg & 1 & 0,9 \\
\hline Schleswig-Holstein & 1 & 0,9 \\
\hline \multicolumn{3}{|l|}{ Bildungsgrad } \\
\hline Hochschulabschluss & 51 & 47,8 \\
\hline (Fach-)Abitur & 37 & 34,2 \\
\hline Realschule (mittlere Reife) & 18 & 16,2 \\
\hline Hauptschulabschluss & 2 & 1,8 \\
\hline \multicolumn{3}{|l|}{ Beschäftigungsart } \\
\hline Angestellt & 102 & 91,9 \\
\hline Selbstständig & 9 & 8,1 \\
\hline \multicolumn{3}{|l|}{ Arbeitszeitmodell } \\
\hline Normalarbeitszeit & 33 & 29,73 \\
\hline Gleitzeit mit Kernzeit & 23 & 20,72 \\
\hline Arbeitszeit auf Vertrauen & 20 & 18,02 \\
\hline Flexible Arbeitszeit & 13 & 11,71 \\
\hline Gleitzeit ohne Kernzeit & 13 & 11,71 \\
\hline Sonstiges & 7 & 6,31 \\
\hline Gleitzeit mit Funktionszeit & 2 & 6,8 \\
\hline Alter & 111 & $M: 30,8$ (SD: 11,46) \\
\hline
\end{tabular}

Anmerkungen: Angaben in Prozent, außer beim Alter $\mathrm{M}=$ Mittelwert; SD = Standardabweichung; Normalarbeitszeit: feste Tages- und Wochenarbeitszeit; Gleitzeit mit Kernzeit: Beginn- und Endzeiten können innerhalb eines gewissen Rahmens variieren, es gibt aber bestimmte Zeiten mit Anwesenheitspflicht; Arbeitszeit auf Vertrauen: keine formalen Arbeitszeitvorgaben; Gleitzeit ohne Kernzeit: Arbeitsbeginn- und Endzeiten können variieren, aber es gibt keine festen Zeiten mit Anwesenheitspflicht; flexible Arbeitszeit: Arbeitszeit kann zwar variieren, wird aber vom Arbeitgeber vorgegeben; Gleitzeit mit Funktionszeit: Abteilung muss immer mindestens von einer Person besetzt sein; $\mathrm{N}=111$.
Vorgesetzten erreichbar“, auf einer Skala von 1 („trifft gar nicht $z u^{\prime \prime}$ ) bis 5 (,trifft völlig zu") bewertet. Aus diesen Werten wird eine Summe gebildet, die den Grad der Entgrenzung der Arbeit abbildet.

Das Ausmaß an suchtartigem Arbeiten wurde mittels der Skala zur Erfassung von Arbeitssucht von Schneider und Bühler (2014) gemessen. Der Test besteht aus 20 dichotomen Items, bspw. „Ich verkürze meine Mittagspause, um länger arbeiten zu können.", welche drei der klassischen Suchtkriterien „Kontrollverlust“, „Dosissteigerung“ und „Entzugserscheinung“ erfassen. Die Items werden bewertet mit "trifft nicht zu“ (Punktwert 0) und "trifft $z u$ “ (Punktwert 1). Bei der Auswertung wird ebenfalls die Summe der erreichten Punktwerte gebildet. Der Gesamtscore gibt Auskunft über das Ausmaß des suchtartigen Arbeitens.

Die Persönlichkeit der Teilnehmer wurde mittels des BigFive-Inventory-SOEP (BFI-S) von Schupp \& Gerlitz (2014) untersucht. Es handelt sich dabei um einen deutschsprachigen Kurztest zur Erfassung der Big Five der Persönlichkeit (Verträglichkeit, Extraversion, Neurotizismus, Gewissenhaftigkeit und Offenheit für Erfahrungen). Er besteht aus 15 Items, die auf einer 7 stufigen Skala $(1=$ „Trifft überhaupt nicht $z u^{\prime \prime} ; 7$ = „Trifft voll $z u^{\text {") }}$ bewertet werden.

Die statistische Auswertung erfolgten mittels der Statistikprogramme „R“ und „PSPP“. Zur Hypothesenprüfung kamen Korrelationsanalysen (Pearson Produkt-Moment Korrelationen), sowie eine moderierte Regressionsanalyse zum Einsatz.

Mittels der Korrelationsanalyse wurden die Zusammenhänge zwischen suchtartigem Arbeiten, Big Five Persönlichkeitsfaktoren, beruflicher Rahmenbedingung (Entgrenzung der Arbeit) und dem Alter der Probanden untersucht. Für die nachfolgende moderierte Regressionsanalyse wurde im ersten Schritt das Alter, im zweiten Schritt der Big Five Persönlichkeitsfaktor Neurotizismus (UV), sowie die Moderatorvariable Entgrenzung der Arbeit und im dritten Schritt noch die Interaktion zwischen Neurotizismus und Entgrenzung zur Vorhersage suchtartigen Arbeitens (Kriterium) aufgenommen. Das Alter wurde in der moderierten Regressionsanalyse, aufgrund des gefundenen signifikanten, korrelativen Zusammenhangs mit suchtartigem Arbeiten, berücksichtigt.

Als abhängige Variable fungierten die Scores des Arbeitssuchtfragebogens von Schneider und Bühler (2014). Die Prädiktoren, sowie die Moderatorvariable wurden vor Durchführung der moderierten Regressionsanalyse mittelwertszentriert.

Um den Zusammenhang zwischen Neurotizismus und suchtartigem Arbeiten getrennt für Probanden mit hohen und niedrigen Entgrenzungswerten darstellen zu können, wurde ein Mediansplit basierend auf der Variablen Entgrenzung angewandt. 
Für die statistischen Tests wurde ein Signifikanzniveau von $\alpha=5 \%$ zugrunde gelegt.

\section{Ergebnisse}

Zur Bestimmung der internen Konsistenz unserer Erhebungsverfahren wurde Cronbachs Alpha für die eingesetzten Messinstrumente berechnet (Tab. 2).

Die Items der Skala zur Erfassung von Arbeitssucht von Schneider und Bühler (2014) und die des Fragebogens zur Erfassung zentraler Merkmale der Arbeit 4.0 können mit einem Cronbachs Alpha von .79 und .80 als intern konsistent bzw. reliabel bezeichnet werden.

Tabelle 2. Interne Konsistenz der verwendeten psychometrischen Tests

\begin{tabular}{lll}
\hline & $\begin{array}{l}\text { Validierungsstudien } \\
\text { Cronbachs Alpha }\end{array}$ & $\begin{array}{l}\text { Eigene Studie } \\
\text { Cronbachs Alpha }\end{array}$ \\
\hline Arbeitssucht & .87 & .79 \\
Entgrenzung & .78 & .80 \\
Neurotizismus & .57 & .77 \\
Verträglichkeit & .53 & .44 \\
Extraversion & .62 & .92 \\
Gewissenhaftigkeit & .72 & .63 \\
Offenheit & .74 & .67 \\
\hline
\end{tabular}

Anmerkungen: Arbeitssucht - Skala zur Erfassung von Arbeitssucht; Entgrenzung - Fragebogen zur Erfassung zentraler Merkmale der Arbeit 4.0; Persönlichkeitsfaktoren - BFI-S; N = 111 .
Cronbachs Alpha für die jeweils drei Items der fünf Persönlichkeitsdimensionen des Big Five Big-Five-InventorySOEP (BFI-S) von Schupp \& Gerlitz (2014) variiert. Die Items der Skalen Neurotizismus, Extraversion und Offenheit sind hinreichend intern konsistent, nicht jedoch die Items zur Erfassung von Verträglichkeit und Gewissenhaftigkeit. Bei der Beurteilung der Werte sollte allerdings beachtet werden, dass die Höhe von Cronbachs Alpha stark von der Itemanzahl abhängt und der kritische Wert für Instrumente mit deutlich mehr Items festgelegt wurde.

Die Ergebnisse der Korrelations- und moderierten Regressionsanalyse sind im Folgenden in den Tabellen 2 bis 4 und Abbildung 1 dargestellt. Die Korrelationsanalysen ergaben signifikante Zusammenhänge zwischen suchtartigem Arbeiten, sowie dem Alter der Teilnehmer $(\mathrm{r}=-.321)$, dem Ausmaß der Entgrenzung der Arbeit $(r=.208)$ und dem Persönlichkeitsfaktor Neurotizismus $(r=.356)$ (siehe Tab. 3). Des Weiteren zeigen die Ergebnisse der Regressionsanalyse, dass das geprüfte Modell inklusive der Variablen Alter, Neurotizismus und Entgrenzung der Arbeit einen signifikanten Erklärungswert im Hinblick auf die Kriteriumsvariable suchtartiges Arbeiten aufweist (F (1, $108)=14.4 ; \mathrm{p}<$.001). Das Alter der Probanden erklärt zehn Prozent der Varianz suchtartigen Arbeitens. Durch Hinzunahme der Variablen Neurotizismus und Entgrenzung der Arbeit erhöhte sich der Anteil der erklärten Varianz um 19 Prozent $\left(\Delta \mathrm{R}^{2}=.19\right)$. Alle drei Prädiktoren zusammen tragen insgesamt 29 Prozent zur Varianzaufklärung bei. Die Daten zeigen allerdings keinen moderierenden Einfluss der Variable Entgrenzung der Arbeit auf den Zusammenhang zwischen der Prädiktorvariablen Neurotizismus und der Kriteriumsvariable suchtartiges Arbeiten (B (SE) = -.01 (.02), $\beta=-.12, \mathrm{t}=-.46, \mathrm{p}=.65)$. Während die Ergebnisse somit Hypothese 1 und 2 stützen,

Tabelle 3. Pearson Korrelationen zwischen suchtartigem Arbeiten, Big 5 Persönlichkeitsfaktoren, beruflicher Rahmenbedingung (Entgrenzung der Arbeit) und Alter

\begin{tabular}{|c|c|c|c|c|c|c|c|c|c|c|}
\hline Variable & M & SD & 1 & 2 & 3 & 4 & 5 & 6 & 7 & 8 \\
\hline Arbeitssucht & 7.38 & 4.15 & - & & & & & & & \\
\hline Entgrenzung & 12.31 & 5.00 & $.208^{\star}$ & - & & & & & & \\
\hline Neurotizismus & 12.65 & 4.09 & $.356 * \star \star$ & .008 & - & & & & & \\
\hline Verträglichkeit & 16.53 & 2.74 & -.151 & .023 & -.165 & - & & & & \\
\hline Extraversion & 14.49 & 3.99 & .065 & -.040 & .054 & .050 & - & & & \\
\hline Gewissenh. & 17.25 & 2.72 & -.086 & -.112 & -.099 & $.203^{\star}$ & -.037 & - & & \\
\hline Offenheit & 15.06 & 3.49 & .018 & .084 & .012 & .052 & .153 & -.020 & - & \\
\hline Alter & 30.8 & 11.46 & $-.321 * \star \star$ & .104 & .001 & .086 & -.159 & -.045 & .009 & - \\
\hline
\end{tabular}

Anmerkungen: Entgrenzung - Fragebogen zur Erfassung zentraler Merkmale der Arbeit 4.0;

Persönlichkeitsfaktoren - BFI-S; Arbeitssucht - Skala zur Erfassung von Arbeitssucht; *p<.05; **p<.01; ***p<.001 (zweiseitig); Gewissenh. = Gewissenhaftigkeit; M = Mittelwert; SD = Standardabweichung; $\mathrm{N}=111$. 
Tabelle 4. Ergebnisse der mittelwertszentrierten Regressionsanalyse zur Vorhersage von suchtartigem Arbeiten.

\begin{tabular}{|c|c|c|c|c|c|}
\hline & $\mathrm{B}$ & SE & $\beta$ & $t$ & $p$ \\
\hline \multicolumn{6}{|l|}{ Variablen } \\
\hline (Konstante) & 7.38 & .34 & .00 & 21.88 & $<.001$ \\
\hline Alter & -.13 & .03 & -.35 & -4.22 & $<.001$ \\
\hline Neurotizismus & .36 & .08 & .35 & 4.35 & $<.001$ \\
\hline Entgrenzung (MOD) & .20 & .07 & .24 & 2.94 & $<.01$ \\
\hline Neurotizismus X Entgrenzung & -.01 & .02 & -.12 & -.46 & .65 \\
\hline \multicolumn{6}{|c|}{ Schritt 1 (Einschluss der Variablen Alter) } \\
\hline$R^{2}$ & .10 & & & & \\
\hline Adj. $R^{2}$ & .09 & & & & \\
\hline SE & 3.95 & & & & \\
\hline$F(1,110)$ & 12.51 & & & & \\
\hline \multicolumn{6}{|c|}{ Schritt 2 (Einschluss der Variablen Neurotizismus und Entgrenzung) } \\
\hline $\mathrm{R}^{2}$ & .29 & & & & \\
\hline Adj. $R^{2}$ & .27 & & & & \\
\hline$\Delta R^{2}$ & .19 & & & & \\
\hline SE & 3.55 & & & & \\
\hline$F(1,108)$ & $14.4 * \star \star$ & & & & \\
\hline \multicolumn{6}{|c|}{ Schritt 3 (Einschluss des Interaktionsterms) } \\
\hline$R^{2}$ & .29 & & & & \\
\hline Adj. $R^{2}$ & .26 & & & & \\
\hline$\Delta \mathrm{R}^{2}$ & 0 & & & & \\
\hline SE & 3.57 & & & & \\
\hline$F(1,107)$ & $10.77 * * \star$ & & & & \\
\hline
\end{tabular}

Anmerkungen: Prädiktoren und Moderator wurden mittelwertszentriert; abhängige Variable = Arbeitssuchtscore gemessen mittels der Skala zur Erfassung von Arbeitssucht, Schneider und Bühler (2014); UV = Unabhängige Variable; $\mathrm{MOD}=$ Moderator; Adj. $\mathrm{R}^{2}=$ korrigiertes $\mathrm{R}^{2} ; \Delta \mathrm{R}=$ Änderung in $\mathrm{R}^{2} ; \mathrm{SE}=\mathrm{Standard}-$ fehler des Schätzers; unstandardisierter Regressionskoeffizient; $\beta=$ standardisierter Regressionskoeffizient; *** $p<.001 ; N=111$.

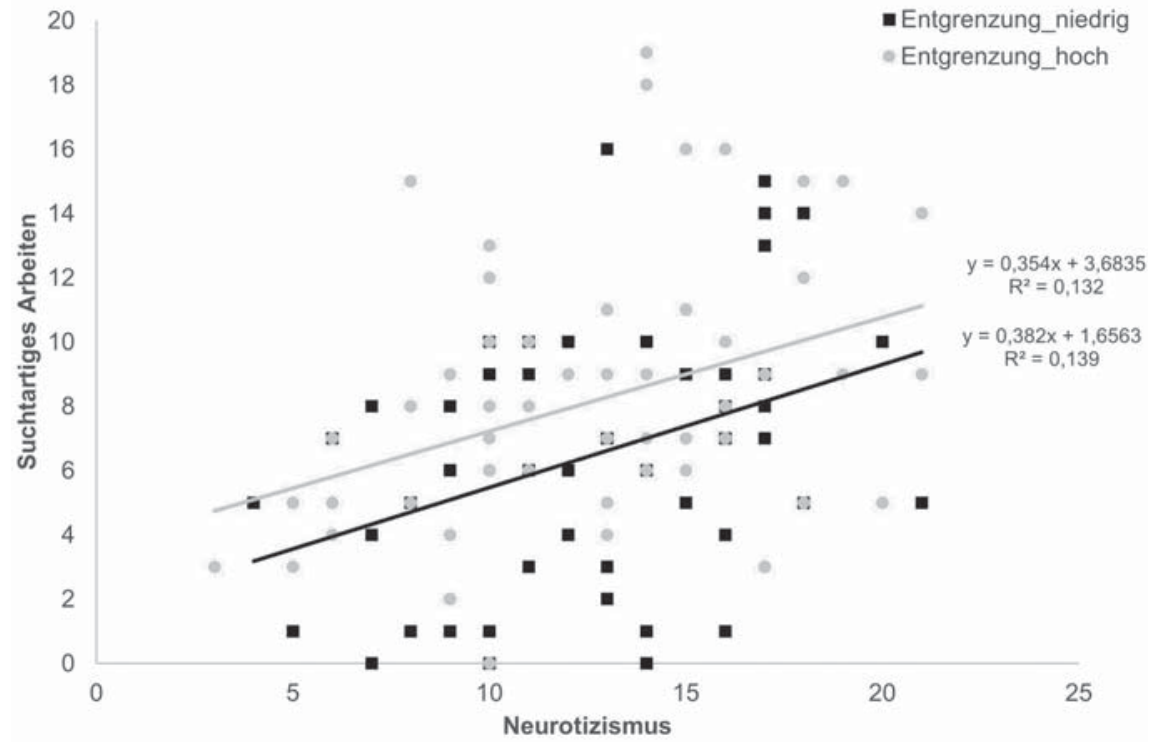

Abbildung 1. Zusammenhang zwischen Neurotizismus und suchtartigem Arbeiten, getrennt dargestellt für Probanden mit hohen und niedrigen Entgrenzungswerten (Mediansplit) 
kann Hypothese 3 mit den vorliegenden Ergebnissen nicht bestätigt werden.

In weiteren Analysen fanden wir außerdem einen schwachen, aber signifikanten Zusammenhang zwischen der Anzahl an geplanten Wochenstunden und Werten im Arbeitssucht-Test $(\mathrm{r}=.214)$. Die tatsächlichen Wochenstunden zeigen mit einer Korrelation von $r=.340$ einen stärkeren Zusammenhang mit suchtartigem Arbeiten als die Menge der geplanten Wochenstunden. Auch zwischen einer zunehmend entgrenzten Arbeit und einem Anstieg der Arbeitszeit fanden wir eine positive Korrelation von $r=.290$.

\section{Diskussion}

Ziel der vorliegenden Untersuchung war es, herauszufinden, welche Rolle berufliche Rahmenbedingungen (Entgrenzung der Arbeit) und Persönlichkeitsfaktoren (Big Five) bei suchtartigem Arbeiten spielen.

Die Ergebnisse zeigten, dass in der untersuchten Stichprobe ein signifikanter Zusammenhang zwischen Neurotizismus und suchtartigem Arbeiten besteht (H1). Das steht im Einklang mit bisherigen Untersuchungen, welche suchtartiges Arbeiten nahe an den klassischen Süchten definieren und diese im Zusammenhang mit Persönlichkeitsfaktoren untersuchen. Weitere Studien berichten nicht nur von einem Zusammenhang zwischen Neurotizismus und suchtartigem Arbeiten, sondern auch mit Sucht allgemein (Homayouni, 2011), mit Videospielabhängigkeit (Mehroof \& Griffiths, 2010) und mit Internetsucht (Senormanci et al., 2014). Im Sinne der Fluchthypothese könnten die gefundenen Zusammenhänge zwischen suchtartigen Verhaltensweisen und dem Persönlichkeitsfaktor Neurotizismus darauf hindeuten, dass sich Menschen übermäßig auf diese Verhaltensweisen einlassen, um unangenehmen Situationen auszuweichen oder eine Konfrontation mit sich selbst oder dem Umfeld zu vermeiden (Andreassen et al., 2016).

Zudem konnte ein signifikanter Zusammenhang zwischen Entgrenzung der Arbeit und suchtartigem Arbeiten gezeigt werden (H2). Da es zu diesem Zusammenhang noch keine konkreten Studien gibt, ist es notwendig, diese Ergebnisse in einer nachfolgenden Untersuchung zu überprüfenUnsere Untersuchung kann nicht beantworten, ob sich Betroffene zunehmend entgrenzte Arbeit suchen oder die entgrenzte Arbeit suchtartiges Arbeiten hervorbringt. Interessant ist hierbei, dass eine zunehmend entgrenzte Arbeit in Zusammenhang mit einer Ausdehnung der Arbeitszeit steht. Dies konnte in dem Maße bestätigt werden, dass in der vorliegenden Stichprobe ein signifikanter Zusammenhang zwischen dem Grad der Entgrenzung der Arbeit und den tatsächlich geleisteten Wochenstunden ge- funden wurde. Dieser Zusammenhang wurde von anderen Forschern bereits vermutet (Poethke et al., 2019). Außerdem konnte gezeigt werden, dass die reine Anzahl an Wochenstunden wiederum im Zusammenhang mit Arbeitssucht steht. Ob diese Mehrarbeit tatsächlich zu einer Erholungsunfähigkeit führt und sich die Betroffenen schwerer gedanklich von der Arbeit lösen können, konnte allerdings nicht beantwortet werden. Weiterführende Untersuchungen sollten diesen Punkt aufgreifen, um mehr über den Zusammenhang und die Ursache-Wirkungs-Beziehung von entgrenzter Arbeit, Arbeitszeit und suchtartigem Arbeiten herauszufinden.

In der dritten Hypothese (H3) wurde getestet, ob der Zusammenhang zwischen dem Persönlichkeitsfaktor Neurotizismus und suchtartigem Arbeiten vom Ausmaß der Entgrenzung der Arbeit beeinflusst (moderiert) wird. Diese Hypothese bildete die konkrete Erweiterung des Modells, da in dieser Arbeit zum ersten Mal suchtartiges Arbeiten nicht nur in Verbindung mit Persönlichkeitsfaktoren, in diesem Fall Neurotizismus, sondern auch mit beruflichen Rahmenbedingungen, in diesem Fall Entgrenzung der Arbeit, untersucht wurde. Die Grundlage für diese Hypothese bildeten die interaktionistischen Theorien, welche die Eigenschaftsmodelle der Persönlichkeit um die Komponente der Situation erweitern (Schmitt \& Altstötter-Gleich, 2010). Unsere Ergebnisse weisen auf einen signifikanten Effekt des Big Five Faktors Neurotizismus und der beruflichen Rahmenbedingung (Entgrenzung der Arbeit) hin, aber wir fanden keine signifikanten Interaktionseffekte. Der Persönlichkeitsfaktor Neurotizismus, das Alter der Personen und die Entgrenzung der Arbeit klären insgesamt $29 \%$ der Varianz bei suchtartigem Arbeiten auf. Die Güte der Vorhersagekraft des statistischen Modells nimmt bei zusätzlicher Berücksichtigung der beruflichen Rahmenbedingung Entgrenzung der Arbeit zu. Dies deutet darauf hin, dass für die Vorhersage suchtartigen Arbeitens sowohl Persönlichkeits- als auch berufliche Rahmenbedingungen eine Rolle spielen. Diese Erkenntnis liegt im Einklang mit einer multikausalen Suchttheorie, welche besagt, dass nie ein Prädiktor allein für eine Suchtentwicklung verantwortlich ist, sondern dass eine Vielzahl an Variablen Einfluss auf die Entstehung und Aufrechterhaltung von Sucht allgemein hat (Poppelreuter, 1997).

Einschränkend muss erwähnt werden, dass die untersuchte Stichprobe nicht repräsentativ für die Erwerbstätigen in Deutschland ist. Die Ergebnisse sollten deshalb in einer repräsentativen Stichprobe an Erwerbstätigen repliziert werden, um eine Generalisierbarkeit der Ergebnisse auf die gesamte Gruppe an Erwerbstätigen in Deutschland zu ermöglichen. Dennoch wurden höhere Werte im Faktor suchtartiges Arbeiten als in der Normstichprobe des Tests erreicht, was im Einklang mit ähnlichen Untersuchungen zu diesem Thema steht. Jüngere Erwerbstätige (18 bis 
45 Jahre) scheinen häufiger suchtartig zu arbeiten als Ältere (46 bis 58 Jahre). Diese Erkenntnis spricht ebenfalls dafür, dass suchtartiges Arbeiten auch durch gesellschaftlichen Wandel vermehrt auftritt (Andreassen et al., 2014) und überwiegend auch jüngere Erwerbstätige von diesem Phänomen betroffen sind. Daher ist es umso wichtiger, einheitliche Kriterien zur Diagnose von suchtartigem Arbeiten bereitzustellen und die klinische Relevanz des Themas stärker in den Mittelpunkt der Diskussion zu rücken. In der Diskussion sind neben einer möglichen Einordnung in die Diagnosekategorie der "Suchterkrankungen“, auch die Zuordnung zu anderen Kategorien wie dem Spektrum der Zwangserkrankungen vorstellbar (Hand, 2003; Sussman, 2012). Jedoch gibt es zunehmend mehr Evidenz, die für eine Einordnung in die Diagnosekategorie der Sucherkrankungen spricht (Andreassen, 2013; Griffiths, Demetrovics \& Atroszko, 2018). Hierzu gibt es auch warnende Stimmen, die vor einer inflationären Verwendung des Suchtbegriffes und einer Pathologisierung der Gesellschaft warnen (Brand et al., 2020; Billieux et al., 2015; Starcevic, Billieux \& Schimmenti, 2018). Brand und Kollegen haben diesbezüglich vorgeschlagen, Kriterien auf der Metaebene zu definieren, die die weitere Erforschung möglicher suchtartiger Verhaltensweisen leiten können. Wenn genügend Hinweise für die klinische Relevanz, theoretische Einbettung und empirische Evidenz für die jeweilige suchtartige Verhaltensweise gefunden werden, könnte diese dann in eine mögliche Kategorie „other specified disorders due to addictive behaviors" im ICD aufgenommen werden. In Bezug auf suchtartiges Arbeiten, existieren Befunde zur klinischen Relevanz und eine theoretische Einbettung in gängige Suchtmodelle ist ebenfalls gegeben, allerdings sollten noch weitere empirische Arbeiten folgen. So existieren zum jetzigen Zeitpunkt kaum Studien zu den neurobiologischen Grundlagen der Arbeitssucht. Wie man am Beispiel anderer Verhaltenssüchte, wie der Glücksspiel- und Computerspielsucht, deren diagnostische Einordnung als Verhaltenssucht zunächst auch umstritten war, sehen kann, erleichtert ein Verständnis der neurobiologischen Grundlagen die diagnostische Einordnung (Fauth-Bühler et al., 2016; Fauth-Bühler \& Mann, 2017; Mann et al., 2016; Kiefer et al., 2013). Ein Vergleich der neurobiolgischen Ähnlichkeiten von anerkannten Verhaltenssüchten und möglichen „neuen“ Verhaltenssüchten erlaubt es, Gemeinsamkeiten und Unterschiede herauszuarbeiten, wie dies z. B. für die Computerspielsucht erfolgt ist (Fauth-Bühler \& Mann, 2017). Denn nur wenn suchtartiges Arbeiten klinisch erfasst ist, können Ärzte eine Diagnose stellen und Patienten, die tatsächlich Hilfe benötigen, adäquat behandelt werden. $\mathrm{Au}-$ ßerdem kann so die Prävalenz von suchtartigem Arbeiten genauer erfasst werden. Dadurch können die gesamtgesellschaftlichen Kosten ermittelt werden und auch einzel- ne Betriebe können die Relevanz der Krankheit für sich besser abschätzen. Die Weiterentwicklungen in DSM-5 und ICD-11- lassen hoffen, dass zukünftig eine einheitliche, diagnostische Möglichkeit zur Erfassung von suchtartigem Arbeiten, wie das für die Computerspielsucht bereits geschehen ist (Saunders et al., 2017) gegeben sein wird.

\section{Schlussfolgerungen für die Praxis}

- Führungskräfte und Mitarbeiter sollten für das Thema suchtartiges Arbeiten sensibilisiert werden.

- Schulungen zum Thema „Suchtartiges Arbeiten“ können helfen, der Entwicklung einer Arbeitssucht vorzubeugen und Betroffenen schneller Hilfe zukommen zu lassen.

Mögliche Themen: gesunder Ausgleich zwischen Arbeit und Freizeit, Zeitmanagementlösungen zur Verhinderung der Ausdehnung von Arbeitszeit, Angebote zur Steigerung der geistigen und körperlichen Fitness.

- Persönlichkeitstests bei Einstellung von neuem Personal können unterstützend von Nutzen sein, um vulnerable Personen zu identifizieren und präventiv agieren $\mathrm{zu}$ können.

- Bei entgrenzter Arbeit sollte zunehmend auf Anzeichen von suchtartigem Arbeiten geachtet werden und Unternehmen sollten eine reine Ausweitung der Arbeitszeit nicht belohnen.

\section{Literatur}

Andreassen, C.S. (2013). Workaholism: An overview and current status of the research. Journal of Behavioral Addictions, 3, 1-11.

Andreassen, C.S., Bjorvatn, B., Moen, B.E., Waage, S., Mageroy, N. \& Pallesen, S. (2016). A longitudinal study of the relationship between the five-factor model of personality and workaholism TPM - Testing, Psychometrics, Methodology in applied psychology, 23, 285-298.

Andreassen, C.S., Griffiths, M.D., Gjertsen, S.R., Krossbakken, E., Kvam, S. \& Pallesen, S. (2013). The relationship between behavioral addictions and the five-factor model of personality. Journal of Behavioral Addicitons, 2, 90-99.

Andreassen, C.S., Griffiths, M.D., Hetland, J., Lravina , L., Jensen, F. \& Pallesen, S. (2014). The Prevelance of Workaholism: A Survey Study in a National Representative Sample of Norwegien Employees. PLOS ONE, 9(8), e102446. https://doi.org/10.1371/ journal.pone.0102446.

Aziz, S. \& Tronzo, C.L. (2011). Exploring the relationship between workaholism facets and personality traits: a replication in american workers. The Psychology Record, 61, 269-286.

Bakker, A.B., Demerouti, E. \& Burke, R. (2009). Workaholism and Relationship Quality: A Spillover-Crossover Perspective. Journal of Occupational Health Psychology, 14, 23-33.

Böhle, F. (2018). Arbeit und Belastung. In F. Böhle, G. G. Voß \& G Wachtler (Hrsg.), Handbuch Arbeitssoziologie - Band 2: Akteu- 
re und Institutionen, 2. Auflage (S. 59-98). Wiesbaden: Springer-Verlag.

Billieux, J., Schimmenti, A., Khazaal, Y., Maurage, P. \& Heeren, A. (2015). Affiliations expand Are we overpathologizing everyday life? A tenable blueprint for behavioral addiction research. Journal of Behavioral Addictions, 4, 119-123.

Brand, M., Rumpf, H.J., Demetrovics, Z., Müller, A., Stark, R., King, D. L. et al. (2020). Which conditions should be considered as disorders in the International Classification of Diseases (ICD-11) designation of "other specified disorders due to addictive behaviors"? Journal of Behavioral Addictions. doi: 10.1556/2006.2020.00035

Burke, R.J., Matthiesen, S.B. \& Pallessen, S. (2006). Personality correlates of workaholism. Personality and Individual Differences, 40, 1223-1233.

Der Spiegel (1958, 11. Juni). Manager-Krankheit - Der chemische Herztod. Der Spiegel, S. 54. Verfügbar unter https://www.spiegel.de/politik/der-chemische-herztod-a-7079870c-00020001-0000-000041762184?context=issue

Fassel, D. (1991). Wir arbeiten uns noch zu Tode - Die vielen Gesichter der Arbeitssucht. München: Kösel-Verlag.

Fauth-Bühler, M. \& Mann, K. (2017). Neurobiological correlates of internet gaming disorder: Similarities to pathological gambling. Addictive Behaviors, 64, 349-356.

Fauth-Bühler, M., Mann, K. \& Potenza, M.N. (2016). Pathological gambling: a review of the neurobiological evidence relevant for its classification as an addictive disorder. Addiction Biology, 22, 885-897.

Griffiths, M.D. (2011). Workaholism: A 21st century addiciton. The Psychologist: Bulletin of the British Psychological Society, 24, 740-744.

Griffiths, M.D., Demetrovics, Z. \& Atroszko, P.A. (2018). Ten myths about work addiction. Journal of Behavioral Addicitons, 7 , 845-857.

Hägerbäumer, M. (2017). Risikofaktor Präsentismus - Hintergründe und Auswirkungen des Arbeitens trotz Krankheit. Wiesbaden: Springer.

Hand, I. (2003). Störungen der Impulskontrolle: Nichtstoffgebundene Abhängigkeiten (Süchte), Zwangsspektrum-Störungen... oder? Suchttherapie, 4, 51-53.

Hassler, M., Rau, R., Hupfeld, J. \& Paridon, H. (2013). Auswirkungen von ständiger Erreichbarkeit und Präventionsmöglichkeiten. Dresden: Zukunft der Arbeit GmbH.

Homayouni, A. (2011). The role of personality traits and religious beliefs in tendency to addiction. Procedia - Social and behavioral sciences, 30, 851-855.

Jackson, S.S., Fung, M.-C., Moore, M.-A.C. \& Jackson, C.J. (2016). Personality and Workaholism. Personality and Individual Differences, 95, 114-120.

Kiefer, F., Fauth-Bühler, M., Heinz, A. \& Mann, K. (2013). Neurobiologische Grundlagen der Verhaltenssüchte. Der Nervenarzt, 84, 557-562.

Kleemann, F. \& Voß, G.G. (2018). Arbeit und Subjekt. In F. Böhle, Voß, G.G. \& Wachtler G. (Hrsg.), Handbuch Arbeitssoziologie Band 2: Akteure und Institutionen, 2. Auflage (S. 15-58). Wiesbaden: Springer.

Lior, O., Abira, R. \& Aviv, W. (2018). Work addiciton: An organizational behavior as well as an addicitive behavior? Journal of behavioral addicitons, 7, 888-891.

Loscalzo, Y. \& Giannini, M. (2017). Clinical conceptualization of workaholism: A comprehensive model. Organizational Psychology Review, 7, 306-329.

Machlowitz, M. (1981). Arbeiten auch Sie zu viel? Landsberg am Lech: Moderne Verlagsgesellschaft mbH.

Mann, K., Fauth-Bühler, M., Higuchi, S., Potenza, M. N., Saunders, J.B. (2016). Pathological gambling: a behavioral addiction. World Psychiatry, 15, 297-298.
Mann, K., Lemenager, T., Zois, E., Hoffmann, S., Nakovics, H., Beutel, M. et al. (2017). Comorbidity, family history and personality traits in pathological gamblers compared with healthy controls. European Psychiatry, 42, 120-128.

Mehroof, M. \& Griffiths, M. D. (2010). Online Gaming Addiction: The Role of Sensation Seeking, Self-Control, Neuroticism, Aggression, State Anxiety and Trait Anxiety. Cyberpsychologie, Behavior and Social Networking, 13, 313-316.

Meißner, E. M. (2005). Die „Droge“Arbeit. Frankfurt am Main: Europäischer Verlag der Wissenschaften.

Mentzel, G. (1979). Über die Arbeitssucht. Zeitschrift für psychosomatische Medizin und Psychoanalyse, 5, 115-127.

Möhring, K., Naumann, E., Reifenscheid, M., Blom, A.G., Wenz, A., Rettig, T. et al. (2020). Die Mannheimer Corona-Studie: Schwerpunktbericht zu Erwerbstätigkeit und Kinderbetreuung (Arbeitspapier). Universtität Mannheim. Verfügbar unter https://madoc. bib.uni-mannheim.de/55139/

Neyer, F.J. \& Asendorpf, J.B. (2018). Psychologie der Persönlichkeit. Berlin: Springer.

Oates, W.E. (1971). Confessions of a workaholic. Nashville: Abingdon Press.

Poethke, U., Klasmeier, K. N., Diebig, M., Hartmann, N. \& Rowold, J. (2019). Entwicklung eines Fragebogens zur Erfassung zentraler Merkmale der Arbeit 4.0. Zeitschrift für Arbeits- und Organisationspsychologie, 63, 129-151.

Poppelreuter, S. (1997). Arbeitssucht. Weinheim: Psychologie Verlags Union.

Rademacher, U. (2017). Arbeitssucht - Workaholismus erkennen und verhindern. Wiesbaden: Springer.

Ruiner, C. \& Wilkesmann, M. (2016). Arbeits- und Industriesoziologie. Paderborn: Wilhelm Fink.

Rump, J. \& Eilers, S. (2017). Arbeit 4.0 - Leben und Arbeiten unter neuen Vorzeichen. In Rump, J. \& Eilers, S. (Hrsg.), Auf dem Weg zur Arbeit 4.0 (S. 3-78). Berlin: Springer.

Saunders, J. B., Hao, W., Long, J., King, D. L., Mann, K., Fauth-Bühler, M. et al. (2017). Gaming disorder: Its delineation as an important condition for diagnosis, management, and prevention. Journal of Behavioral Addictions, 6, 271-279.

Schmitt, M. \& Altstötter-Gleich, C. (2010). Differentielle Psychologie und Persönlichkeitspsychologie. Weinheim: Beltz Verlag.

Schneider, C. \& Bühler, K.-E. (2014). Skala zur Erfassung von Arbeitssucht. Zusammenstellung sozialwissenschaftlicher Items und Skalen. doi:10.6102/zis90.

Schupp, J. \& Gerlitz, J.-Y. (2014). Big Five Inventory-SOEP (BFI-S). Zusammenstellung sozialwissenschaftlicher Items und Skalen. doi: 10.6102/zis54.

Senormanci, Ö., Saraçlı, Ö., Atasoy, N., Șenormancı, G., Koktürk, F. \& Atik, L. (2014). Relationship of Internet addiction with cognitive style, personality, and depression in university students. Comprehensive Psychiatry, 55, 1385-1390.

Sharma, J. \& Sharma, P. (2011). Workaholism and its correlates: A study of academicians. International journal of management and business research, 1, 151-160.

Součková, M., Vaculík, M. \& Procházka, J. (2014). Personality Traits and workaholism. International Journal of Humanities and Social Science, 4, 70-79.

Starcevic, V., Billieux, J. \& Schimmenti, A. (2018). Selfitits, selfie addiction, Twitteritis: Irresistible appeal of medical terminology for problematic behaviours in the digital age. Australien \& New Zealand Journal of Psychiatry, 52, 408-409.

Städele, M. \& Poppelreuter, S. (2009). Arbeitssucht - Neuere Erkenntnisse in Diagnose, Intervention, Prävention. In D. Batthyány, \& A. Pritz (Hrsg.), Rausch ohne Drogen - Substanzungebundene Süchte (S. 141-161). Wien: Springer.

Statistisches Bundesamt (Destatis) (2018a). Reihe 4.1 Erwerbsbeteiligung der Bevölkerung. Wiesbaden: Autor. Verfügbar unter 
https://www.destatis.de/DE/Service/Bibliothek/_publikatio nen-fachserienliste-1.html

Statistisches Bundesamt (Destatis) (2018b). Arbeitsmarkt. In Statistisches Jahrbuch - Deutschland und Internationales (S. 353384). Wiesbaden: Autor.

Statistisches Bundesamt (2019a). Pressemitteilung Nr. 289 vom 31. Juli 2019. Juni 2019: 0,9\% mehr Erwerbstätige als im Vorjahresmonat. Wiesbaden: Autor.

Statistisches Bundesamt (2019b). Erwerbsbeteiligung. Wiesbaden: Autor. Verfügbar unter https://www.destatis.de/DE/Themen/Arbeit/Arbeitsmarkt/Erwerbstaetigkeit/Tabellen/er werbstaetige-erwerbstaetigenquote.html\#fussnote-2-103716

Sussman, S. (2012). Workaholism: A Review. Journal of Addiction Research \& Therapy, 6, 4120.

Weimar, R., Braakmann, D., Gelo, O. \& Schiava-Winkler, U. (2009). Arbeitssucht und Hochleistung. In D. Batthyany \& A. Pritz (Hrsg.), Rausch ohne Drogen - substanzungebundene Süchte (S. 163-190). Wien: Springer.

Zilberman, N., Yadid, G., Efrati, Y., Neumark, Y. \& Rassovsky, Y. (2018). Personality profiles of substance and behavioral addictions. Addictive Behaviors, 82, 174-181.

\section{Historie}

Manuskript eingereicht: 01.12.2020

Manuskript angenommen: 29.03.2021

\section{Deklaration konkurrierender Interessen}

Die Autoren deklarieren keine konkurrierenden Interessen.

\section{Förderung}

Open-Access-Förderung ermöglicht durch die FOM Hochschule für Oekonomie \& Management, Stuttgart.

\section{ORCID}

Mira Fauth-Bühler

(D) https://orcid.org/0000-0002-4182-2049

\section{Prof. Dr. Mira Fauth-Bühler}

FOM Hochschule für Oekonomie \& Management Institut für Wirtschaftspsychologie (iwp)

Hochschulzentrum Stuttgart

Rotebühlstraße 121

70178 Stuttgart

Deutschland

mira.fauth-buehler@fom.de 